\title{
GENERALIZATIONS OF CESȦRO CONTINUOUS FUNCTIONS AND INTEGRALS OF PERRON TYPE
}

BY

\author{
CHENG-MING LEE
}

\begin{abstract}
The linear space of all the Cesaro continuous functions of any order is extended by introducing pointwisely Cesàro continuous functions and exact generalized Peano derivatives. Then six generalized integrals of Perron type are defined and studied. They are based on three recent monotonicity theorems and each depends on an abstract upper semilinear space of certain functions. Some of the integrals are more general than all the integrals in the Cesaro-Perron scale provided that the abstract semilinear space is taken to be the linear space of all the pointwisely Cesaro continuous functions or all the exact generalized Peano derivatives. That such a concrete general integral is possible follows from the fact proved here that each exact generalized Peano derivative is in Baire class one and has the Darboux property. Relations between the pointwisely Cesaro continuous functions or the exact generalized Peano derivatives and functions defined by means of the values of certain Schwartz's distributions at "points" are also established.
\end{abstract}

0. Introduction. The integrals in the Cesàro-Perron scale of integration defined by J. C. Burkill [12] have been studied and generalized by many authors. For examples, see [2], [13], [16]-[26], [28], [29], [37], [39]-[41], [43], [44]. Although each integral in the scale has been generalized in many different ways, no single integral among those known concrete generalized integrals is more general than all the integrals in the scale. To obtain such an integral, we are led to consider not only the class of all the functions which are Cesàro continuous of some order, but also the pointwisely Cesàro continuous functions (Definition 1 in $\$ 2$ ) and the "generalized" Peano derivatives (Definition 2 in \$2). Relations between these two concepts are stated as corollaries after Definition 2 . Roughly speaking, a pointwisely Cesàro continuous function is an exact generalized Peano derivative.

In order to use the exact generalized Peano derivatives to define integrals which are more general than all the integrals in the Cesàro-Perron scale, we prove that each exact generalized Peano derivative is in Baire class one and has the Darboux property (Theorem 2 and Theorem 3 in \$2). It seems interesting to note that the concept of generalized Peano derivatives is closely related to the concept of the values of Schwartz's distributions at "points" due to Kojasiewicz in [31]. A distribution on the real line is not a function defined on the real line but is a linear

Received by the editors March 31, 1980 and, in revised form, June 18, 1980.

1980 Mathematics Subject Classification. Primary 26A39, 26A24; Secondary 26A21.

Key words and phrases. Cesàro-Perron integrals, Denjoy integrals, Cesàro continuous functions, exact Peano derivatives, approximate derivatives, symmetric derivatives, approximate symmetric derivatives, Baire class one, Darboux property, values of distributions of points, pointwisely Cesàro continuous functions, exact generalized Peano derivatives. 
functional defined on the space of test functions. However, the class of all distributions which have values at every point of the real line in Kojasiewicz's sense can be identified with a class of functions defined and finite everywhere on the real line. This class of functions happen to be "just" that of all the exact generalized Peano derivatives (see Theorem 4 and its corollaries in \$2).

To develop the theory of integrals, at the beginning of $\$ 3$ we quote three monotonicity theorems, respectively due to Bruckner [4], Weil [46] and Lee [29]. Then corresponding to each upper semilinear space $u L$ of certain functions and based on the three monotonicity theorems, six generalized integrals of Perron type, denoted as LPG $(k)$-integral for $k=1,2,3,4,5,6$, are defined and briefly studied. If the functions in the space $u L$ have the Darboux property, then the linear space of all the functions $F$ which are $[A C G]$ and such that both $F$ and $-F$ are in $u L$ is then a $P^{*}$-class as defined by Ellis in [18]. In this case, the LPG(1)-integral here is more general than the resulting $P^{*}$-integral of descriptive Denjoy type as defined by Ellis [18]. These two integrals are in fact equivalent provided that the space $u L$ is closed under uniform convergences. For these results, see Remark 3, Theorems $(6,1)$ and $(7,1)$.

The $\operatorname{LPG}(k)$-integral for $k=1,2,3$ are all related to the "generalized absolutely continuous functions." The only difference in these three integrals is that the differential operators involved are respectively the ordinary approximate derivates for $k=1$, the approximate symmetric derivates for $k=2$, and the ordinary symmetric derivates for $k=3$. Under a minor condition on the space $u L$, the LPG(1)- and LPG(2)-integrals are equivalent while the LPG(3)-integral is strictly less general than the LPG(1)-integral (see Theorem 8, and Corollary 2 to Theorem 9).

The definitions of the $\operatorname{LPG}(k)$-integrals for $k=4,5,6$ are not explicitly related to the "generalized absolutely continuous functions." However, we know that the LPG(4)-integral is somehow related to such functions provided that the space $u L$ is restricted to contain only approximately continuous functions (see Theorem 11, Remarks 5 and 6, Theorem $\mathrm{G}$ and its corollary).

Concrete examples of the LPG $(k)$-integrals are obtained by taking $u L$ to be one of the spaces discussed in $\$ 2$. A brief discussion of these concrete integrals are given and listed as (a) to (f). Note that some of the concrete integrals thus obtained are known and some are new. In particular, the LPG(5)- and LPG(6)-integrals seem to be first introduced here even when the space $u L$ is taken to be the space of all continuous functions. Further properties of these two integrals will be studied in a separate paper. It should also be mentioned that if $u L$ is taken to be the space of all the pointwisely Cesàro continuous functions or the exact generalized Peano derivatives, then the resulted LPG(1)-integral is more general than all the integrals in the Cesàro-Perron scale (see the discussion (e)).

As some of the properties of the LPG $(k)$-integrals. depend on whether the space $u L$ is closed under uniform convergences, the paper ends with a result which shows that the space of all the pointwisely Cesàro continuous functions has this property (Theorem 12). 
For convenience, the definitions of the Cesàro-Perron integrals and the exact Peano derivatives are given in $\S 1$, where an essential relation between the $n$th Cesàro continuous functions and the exact $n$th Peano derivatives is reviewed (Theorem A).

Note that all the functions concerned are (extended-) real-valued functions of a real variable unless it is otherwise stated. Notations used here are chosen to be somewhat explanatory. For terminologies which are not explicitly explained, we refer to the book by Saks [38] and the book by Bruckner [5].

We end this introduction by remarking that "the $A$-integral is more general than the $B$-integral" means "every function which is $B$-integrable is also $A$-integrable to the same value," and "two integrals are equivalent" means "each of the two is more general than the other." The meaning that one integral is strictly more general than the other is then also clear.

1. The Cesàro-Perron integrals and exact Peano derivatives. The integrals in the Cesàro-Perron scale defined by J. C. Burkill ([11], [12]) are denoted as $C_{n} P$-integral for $n=0,1,2, \ldots$ The $C_{0} P$-integral is the classical Perron integral (see Remark 1 below). The $C_{n} P$-integral for $n \geqslant 1$ is defined inductively as follows:

(A) For a function which is $C_{n-1} P$-integrable on an interval $[a, b]$, the $n$th Cesàro mean of $F$ on $[a, b]$, denoted as $C_{n}(F ; a, b)$, is defined to be

$$
n(b-a)^{-n} \int_{a}^{b}(b-t)^{n-1} F(t) d t
$$

where the integral being the $C_{n-1} P$-integral.

(B) A function $F$ is said to be $C_{n}$-continuous at a point $x$ if the function $F$ is $C_{n-1} P$-integrable on a neighborhood of $x$ and is finite at $x$ such that

$$
\lim _{h \rightarrow 0} C_{n}(F ; x, x+h)=F(x) .
$$

The one-sided $C_{n}$-continuities are defined in the obvious manner. If a function $F$ is $C_{n}$-continuous at every point of a set, we simply say that $F$ is $C_{n}$-continuous on the set. When the domain of a function is restricted to a compact interval, the $C_{n}$-continuity (as well as many of the concepts to be considered later on in this paper) of the function at each of the end points of the interval is understood to be one-sided in the usual manner.

(C) For a function $F$ which is finite at the point $x$ and is $C_{n-1} P$-integrable on a neighborhood of $x$, the lower $n$th Cesàro derivate of $F$ at $x$, denoted as $l C_{n} D F(x)$, is defined to be

$$
\liminf _{h \rightarrow 0}(n+1) h^{-1}\left[C_{n}(F ; x, x+h)-F(x)\right] .
$$

The upper $n$th Cesàro derivate, $u C_{n} D F(x)$, is the lim sup of the same "difference" quotient. If $l C_{n} D F(x)=u C_{n} \dot{D} F(x)$, the common value, denoted as $C_{n} D F(x)$, is called the $n$th Cesàro derivative of $F$ at $x$. If $C_{n} D F(x)$ exists and is finite, the function $F$ is said to be $n$th Cesàro differentiable at $x$.

(D) Suppose that $f$ is a function which is defined and finite almost everywhere on a compact interval $[a, b]$. A function $M$ defined on $[a, b]$ is called a $C_{n} P$-major 
function of $f$ on $[a, b]$ if $M(a)=0, M$ is $C_{n}$-continuous on $[a, b]$ and if the following two conditions hold:

( $\alpha) l C_{n} D M(x)>-\infty$ for nearly all $x$ in $[a, b]$;

( $\beta) l C_{n} D M(x) \geqslant f(x)$ for almost all $x$ in $[a, b]$.

A function $m$ is a $C_{n} P$-minor function of $f$ on $[a, b]$ if $-m$ is a $C_{n} P$-major function of $-f$ on $[a, b]$. The function $f$ is said to be $C_{n} P$-integrable on $[a, b]$ if it has both $C_{n} P$-major and minor functions on $[a, b]$ and if

$$
\inf M(b)=\sup m(b),
$$

where the inf is taken over all the $C_{n} P$-major functions $M$ of $f$ on $[a, b]$ and the sup over all the minor functions $m$. In this case, the common value in the above equality is called the $C_{n} P$-integral of $f$ on $[a, b]$ and is denoted as

$$
\left(C_{n} P\right) \int_{a}^{b} f(x) d x \text {. }
$$

(Note that "nearly all $x$ " in $(\alpha)$ means "for all $x$ except possibly on a set of countably many points.")

RemarK 1. Taking $C_{0}(F ; a, b)=F(b)$, the definitions in (B) to (E) make sense even for $n=0$. In fact, the $C_{0}$-continuity is the ordinary continuity, and the $C_{0} D$-derivates and derivatives are the ordinary ones, and the $C_{0} P$-integral is equivalent to the Perron integral mentioned at the beginning of this section. It should also be noted that the definition of the $C_{n} P$-integral here is a little different from that originally given by Burkill [12]. But the two definitions are equivalent (cf. [3]).

Remark 2 . The $C_{n} P$-integral has been further investigated by many authors. See, for example, [2], [3], [7], [9], [13], [17], [19]-[24], [39]-[41], [44]. It should be pointed out that a defect in [12] has been corrected by Verblunsky in [43], and another defect in [39] also has been corrected by Verblunsky in [44].

Now, we turn to the notion of Peano derivatives. Let $F$ be a function which is defined and finite in a neighborhood of a point $x$, and let $l F_{1}(x)$ and $u F_{1}(x)$ denote the lower and upper derivates of $F$ at $x$, respectively, i.e.

$$
l F_{1}(x)=\liminf _{h \rightarrow 0}[F(x+h)-F(x)] / h,
$$

and similarly for $u F_{1}(x)$. If $l F_{1}(x)=u F_{1}(x)$, the common value denoted by $F_{1}(x)$ is of course the ordinary derivative of $F$ at $x$ and may be finite or infinite. In case $F_{1}(x)$ is finite, the function $F$ is said to be (1st Peano) differentiable at $x$. Inductively, for $k \geqslant 2$, assuming that $F_{1}(x), F_{2}(x), \ldots, F_{k-1}(x)$ exist and are finite, the lower $k$ th Peano derivate of $F$ at $x$ is defined as

$$
l F_{k}(x)=\lim \inf \frac{k !}{h^{k}}\left[F(x+h)-F(x)-\sum_{i=1}^{k-1} \frac{h^{i}}{i !} F_{i}(x)\right],
$$

and similarly for $u F_{k}(x)$. If $l F_{k}(x)=u F_{k}(x)$, the common value, denoted by $F_{k}(x)$, is called the $k$ th Peano derivative of $F$ at $x$. If $F_{k}(x)$ is finite, the function $F$ is said to be $k$ th Peano differentiable at $x$. 
If the function $F$ is $k$ th Peano differentiable at every point of an interval, then the $k$ th Peano derivative, $F_{k}$, is itself a finite function on the interval, and is called an exact $k$ th Peano derivative on the interval.

If in the definitions of the Peano derivates and derivatives, one replaces the ordinary lim inf and lim sup by the approximate lim inf and lim sup, one has the definitions of approximate Peano derivates and derivatives. Both the exact Peano and exact approximate Peano derivatives share many interesting properties with the ordinary derivatives. For example, such exact derivatives have the Darboux property and are in Baire class one (cf. [35], [15], and [1]). Further interesting properties can be found in [45], [43], [27], [36], [30].

As studied by Bergin in [2] (cf. also [28]), the $C_{n} P$-integral can be characterized by directly using Peano derivates. In fact, this can be seen easily from the following

TheOREM A (CF. [2] OR [28]). For a function $M$ to be $C_{n}$-continuous on a compact interval $I$ it is necessary and sufficient that $M$ is an exact nth Peano derivative on $I$. Furthermore, if $M$ is the exact nth Peano derivative of a function $F$ on $I$, then $l C_{n} D M(x)=l F_{n+1}(x)$ and $u C_{n} D M(x)=u F_{n+1}(x)$ for all $x$ in $I$.

The following lemma, to be used later, is essential in the proof of the necessary part of Theorem A.

Lemma 1. Let $M$ be $C_{n-1} P$-integrable on $[a, b]$. Then

$$
C_{n}(M ; x, x+h)=\frac{n !}{h^{n}}\left\{F(x+h)-F(x)-\sum_{i=1}^{n-1} \frac{h^{i}}{i !} F^{i}(x)\right\}
$$

for all $x \in[a, b]$ and for all $h$ with $x+h \in[a, b]$, where

and $F=F^{0}$.

$$
\begin{aligned}
F^{n-1}(x) & =\left(C_{n-1} P\right) \int_{a}^{x} M(t) d t, \\
F^{i}(x) & =\left(C_{k} P\right) \int_{a}^{x} F^{i+1}(t) d t \quad \text { for } 0<i<n-1
\end{aligned}
$$

Proof. This follows from the integration by parts formula (see Theorem 5 in [12]) for the $C_{k} P$-integral for $k=n-1, n-2, \ldots, 3,2,1$.

2. Pointwisely Cesàro continuous functions and generalized Peano derivatives. For each interval $I$ and for each nonnegative integer $n$, denote by $C_{n}\left(=C_{n}(I)\right)$ the class of all the functions which are defined and $C_{n}$-continuous on $I$, and let

$$
C_{\infty}=\bigcup_{n=0}^{\infty} C_{n} \text {. }
$$

Then, it follows from the theory (see [12]) of the scale of Cesàro-Perron integrals that one has

$$
C_{n} \subsetneq C_{n+1} \subsetneq C_{\infty},
$$

and each of the classes $C_{n}$ and $C_{\infty}$ forms a linear space under the usual linear operations of functions. Functions in $C_{\infty}$ are called Cesàro continuous (or simply $C_{\infty}$ ) functions on $I$. Thus, a function is Cesàro continuous on $I$ if and only if there 
exists a nonnegative integer $n$ such that the function is $C_{n}$-continuous at every point of the interval $I$. Allowing the number $n$ to depend not only on the function but also on the point of the interval, we consider a pointwise extension of the $C_{\infty}$ function as follows:

Definition 1. A function defined on the interval $I$ is said to be pointwisely Cesàro continuous on $I$ if for each point $x$ in $I$ there exists a positive integer $n$ $(=n(x))$ such that the function is $C_{n}$-continuous at $x$.

Let us denote by $p C_{\infty}$ the class of all pointwisely Cesàro continuous functions on I. Then it is clear that $p C_{\infty}$ is a linear space containing the space $C_{\infty}$. Since the set $\{n(x): x \in I\}$ in Definition 1 is not necessarily bounded, one concludes that $p C_{\infty} \supsetneq C_{\infty}$. Then, by Theorem $\mathrm{A}$ in the previous section, a pointwisely Cesàro continuous function is not necessarily an exact Peano derivative. However, the following result shows that it is "almost" an exact Peano derivative.

THEOREM 1. Let $f$ be a pointwisely Cesàro contiuous function on the interval 1 . Then for each compact interval $J \subseteq I$ there exist a nonnegative integer $n$ and a continuous function $F$ such that the $(n+1)$ th Peano derivative of $F$ exists and is equal to $f$ almost everywhere on $J$. Furthermore for every $x$ in $J$ there exists a nonnegative integer $n(x) \geqslant n$ such that $G_{n(x)+1}(x)$ exists and is equal to $f(x)$, where $G$ is an $(n(x)-n)$ th fold Riemann indefinite integral of the continuous function $F$ on $J$, (the 0 th fold indefinite integral of a continuous function is understood as the continuous function itself plus a constant).

Proof. For each $x \in I$, let $n(x)$ be a nonnegative integer such that the function $f$ is $C_{n(x)+1}$-continuous at $x$. Then for each $x \in J$ there exists an interval $J(x)$, open in $I$ and containing the point $x$, such that the function $f$ is $C_{n(x)} P$-integrable on the closure of $J(x)$. Since the interval $J$ is compact, there exists a finite set $\left\{x_{1}, x_{2}, \ldots, x_{k}\right\} \subseteq J$ such that $\cup_{i=1}^{k} J\left(x_{i}\right) \supseteq J$. Then it follows easily from the consistency (see [12]) of the scale of the Cesàro-Perron integrals that the function is $C_{n} P$-integrable on $J$ for every integer $n$ which is greater or equal to every $n\left(x_{i}\right)$ for $i=1,2,3, \ldots, k$. Let such an $n$ be fixed, and let $M$ be an indefinite $C_{n} P$-integral of the function $f$, say

$$
M(x)=\left(C_{n} P\right) \int_{a}^{x} f(t) d t \text { for } x \text { in } J=[a, b] .
$$

Then (see [12]) $M$ is $C_{n}$-continuous on $J$ and furthermore

$$
C_{n} \operatorname{DM}(x)=f(x)
$$

for almost all $x$ in $J$. As $M$ is $C_{n}$-continuous on $J$, it follows from Theorem A that there exists a continuous function $F$ such that $M$ is the exact $n$th Peano derivative of $F$ on $J, l C_{n} D M(x)=l F_{n+1}(x)$ and $u C_{n} D M(x)=u F_{n+1}(x)$ for all $x$ in $J$. The last three equalities imply that

for almost all $x \in J$.

$$
F_{n+1}(x)=C_{n} D M(x)=f(x)
$$

It should be noted that the function $F$ above is not unique. For convenience, the $F$ is taken as the one given in Lemma 1, which has all the properties stated in the last paragraph. 
Now, let $x \in J$. Since $f$ is $C_{n(x)+1}$-continuous at $x$, it is also $C_{m+1}$-continuous at $x$ for all integers $m \geqslant n(x)$. Thus, if $n(x) \leqslant n$, we see that $f$ is $C_{n+1}$-continuous at $x$, so that it follows from Lemma 1 that

$$
\begin{aligned}
f(x) & =\lim _{h \rightarrow 0} C_{n+1}(f ; x, x+h) \\
& =\lim _{h \rightarrow 0} \frac{(n+1) !}{h^{n+1}}\left\{F(x+h)-F(x)-\sum_{i=1}^{n} \frac{h^{i}}{i !} F^{i}(x)\right\} \\
& =F_{n+1}(x),
\end{aligned}
$$

where $F^{n}=M$ on $J$. If $n(x)>n$ then $F$ may not be $(n+1)$ th Peano differentiable at $x$; but then by Lemma 1 the $C_{n(x)+1}$-continuity of $f$ at $x$ implies that

$$
\begin{aligned}
f(x) & =\lim _{h \rightarrow 0} C_{n(x)+1}(f ; x, x+h) \\
& =\lim _{h \rightarrow 0} \frac{(n(x)+1) !}{h^{n(x)+1}}\left\{G(x+h)-G(x)-\sum_{i=1}^{n(x)} \frac{h^{i}}{i !} G^{i}(x)\right\} \\
& =G_{n(x)+1}(x),
\end{aligned}
$$

where

$$
\begin{aligned}
G^{n(x)}(y) & =\left(C_{n(x)} P\right) \int_{a}^{y} f(t) d t, \\
G^{i}(y) & =\left(C_{i} P\right) \int_{a}^{y} G^{i+1}(t) d t \quad \text { for } 0<i<n(x),
\end{aligned}
$$

and $G(y)=G^{0}(y)$. Then, by the consistency of the scale of the Cesàro-Perron integrals again, we have $G^{n(x)}=M$ and $G^{n(x)-n}=F$ on $J$. Since $F$ is continuous on $J$, the function $G=G^{0}$ is then just a $(n(x)-n)$ th fold Riemann integral of $F$ on $J$. The proof is hence completed.

Motivated by Theorem A and Theorem 1, we give another "pointwise" extension of $C_{\infty}$ functions in the following definition. For convenience, if $F$ is a continuous function on an interval, we will write $F^{-0}=F^{0}=F$ on the interval and for $k \geqslant 1$, $F^{-k}(x)=\int^{x} F^{-(k-1)}(t) d t$, an indefinite integral of $F^{-(k-1)}$ on the interval. Thus, $F^{-k}$ is a $k$ th fold Riemann integral of the continuous function $F$.

Definition 2. Let $n$ be a positive integer. A function $F$ is said to be generalized $n$th Peano differentiable at the point $x$ if the function $F$ is continuous in a neighborhood of $x$ and if there exists a nonnegative integer $k$ such that $F^{-k}$ is $(n+k)$ th Peano differentiable at $x$; in this case the $(n+k)$ th Peano derivative of $F^{-k}$ at $x$, denoted as $F_{[n]}(x)$, is called the generalized $n$th Peano derivative of $F$ at $x$. (Note that the number $F_{[n]}(x)$ is independent of the integer $k$ whenever the function $F$ is generalized $n$th Peano differentiable at $x$.) If $F_{[n]}(x)$ exists for all $x$ in an interval, the finite function $F_{[n]}$ is said to be an exact generalized $n$th Peano derivative on the interval.

Let $G_{n} P D\left(=G_{n} P D(I)\right)$ be the class of all the exact generalized $n$th Peano derivatives on $I$, and denote $G P D=\cup_{n=1}^{\infty} G_{n} P D$. Then, clearly each of the $G_{n} P D$ 
and $G P D$ is a linear space. Furthermore, Theorem 1 gives rise to the following corollaries:

COROllary 1. If $f$ is a pointwisely Cesàro continuous function on an interval, then for each compact interval $J \subseteq I$ there exist a positive integer $n$ and a continuous function $F$ such that $F_{[n]}(x)=f(x)$ for all $x$ on $J$, and for almost all $x$ in $J$ the generalized Peano derivative $F_{[n]}(x)$ is just the ordinary one $F_{n}(x)$.

COROLlaRY 2. Every pointwisely Cesàro continuous function on a compact interval is an exact generalized Peano derivative there.

We conjecture that neither the converse of Corollary 2 nor the converse of Corollary 1 holds true.

We generalize the fact that each exact Peano derivative is in the class of Baire one and has the Darboux property (cf. Oliver [35]) with the following results:

TheOREM 2. Each exact generalized Peano derivative on an interval is in Baire class one.

Theorem 3. Each exact generalized Peano derivative on an interval has the Darboux property there.

Proof of Theorem 2. Let $F$ be a continuous function which is generalized $n$th Peano differentiable on the interval $I$. It is required to show that $F_{[n]}$ is in the class of Baire one on $I$. To this end, let $x_{0} \in I$ be fixed. There exists a nonnegative integer $k=k\left(x_{0}\right)$ such that $F^{-k}$ is $(n+k)$ th Peano differentiable at $x_{0}$. Writing $G=F^{-k}$, and

$$
Q(x)=Q\left(x, x_{0}\right)=G(x)-G\left(x_{0}\right)-\sum_{i=1}^{n+k-1} \frac{\left(x-x_{0}\right)^{i}}{i !} G_{i}\left(x_{0}\right),
$$

one has

$$
F_{[n]}\left(x_{0}\right)=\lim _{x \rightarrow x_{0}} \frac{(n+k) !}{\left(x-x_{0}\right)^{n+k}} Q(x)
$$

Then one sees that

$$
\lim _{a \rightarrow 0} \frac{Q\left(a u+x_{0}\right)}{a^{n+k}}=\frac{u^{n+k}}{(n+k) !} F_{[n]}\left(x_{0}\right)
$$

uniformly for $u$ in any compact interval.

Now, let us take $\phi$ to be an infinitely differentiable function with compact support and $\int_{-\infty}^{\infty} \phi(t) d t=1$. It follows from (1) that we have

$$
\lim _{a \rightarrow 0} \int_{-\infty}^{\infty} \frac{Q\left(a u+x_{0}\right)}{a^{n+k}} \phi^{(n+k)}(u) d u=F_{[n]}\left(x_{0}\right) \int_{-\infty}^{\infty} \frac{u^{n+k}}{(n+k) !} \phi^{(n+k)}(u) d u .
$$

Then integration by parts yields the following:

$$
\lim _{a \rightarrow 0} \int_{-\infty}^{\infty} \frac{G\left(a u+x_{0}\right)}{a^{n+k}} \phi^{(n+k)}(u) d u=(-1)^{n+k} F_{[n]}\left(x_{0}\right)
$$


Note that the function $G$ is at least $k$ times differentiable and its $k$ th derivative is the continuous function $F$. Hence, by integration by parts again, one has

$$
\lim _{a \rightarrow 0} H_{a}\left(x_{0}\right)=F_{[n]}\left(x_{0}\right)
$$

where

$$
\begin{aligned}
H_{a}\left(x_{0}\right) & =\int_{-\infty}^{\infty} \frac{F\left(a u+x_{0}\right)}{(-1)^{n} a^{n}} \phi^{(n)}(u) d u \\
& =\int_{-\infty}^{\infty} \frac{F(v)}{(-1)^{n} a^{n}|a|} \phi^{(n)}\left(\frac{v-x_{0}}{a}\right) d v
\end{aligned}
$$

Therefore $H_{a}\left(x_{0}\right)$ is a continuous function of $x_{0}$ on $I$, and hence $F_{[n]}$ is in the class of Baire one on I, completing the proof.

The proof of Theorem 2 given above is quite different from that known for the Peano derivative (cf. [31]), and is closely related to Schwartz's distribution theory [42]. In fact, the proof arises from a study of the interesting paper [31] by Kojasiewicz, and is given here to show a flavor of the generalized notions involved. We will not give a direct proof of Theorem 3 here. Instead, we will review a little bit about Kojasiewicz's work and indicate that both Theorem 2 and Theorem 3 can be obtained from his work.

A distribution $T$ on the real line is said (see [31]) to have a value at a point $x_{0}$ on the real line if the distribution $T\left(a x+x_{0}\right)$ converges in the distributional sense as $a \rightarrow 0$, i.e. for each test function $\phi$,

$$
\left\langle T, \frac{1}{|a|} \phi\left(\frac{x-x_{0}}{a}\right)\right\rangle \text { converges as } a \rightarrow 0 .
$$

Note that if $T\left(a x+x_{0}\right)$ converges in the distributional sense as $a \rightarrow 0$, then the limit is a constant distribution, and this constant is called the value of the distribution at the point $x_{0}$ and will be denoted as $v\left(T, x_{0}\right)$. Kojasiewicz showed that a distribution is uniquely determined by its values provided that they exist everywhere (see Corollary 3 to Theorem 5.2 in [31]). Among others, he also has established the following results:

Theorem B (Theorem 2.3 In [31]). For a distribution $T$ to have value, $v\left(T, x_{0}\right)$, at $x_{0}$ it is necessary and sufficient that there exist a function $F$ which is continuous in $a$ neighborhood of $x_{0}$ and a nonnegative integer $n$ such that the nth distributional derivative of $F$ is equal to the distribution $T$ on the neighborhood of $x_{0}$ and

$$
\lim _{x \rightarrow x_{0}} \frac{F(x)}{\left(x-x_{0}\right)^{n}}=\frac{v\left(T, x_{0}\right)}{n !} .
$$

TheOREM C (THEOREM 5.4 IN [31]). If the value, $v(T, x)$, of a distribution $T$ exists for all $x$, then $v(T, x)$ as a function of $x$ is in the class of Baire one and has the Darboux property. 
Theorem 2 and Theorem 3 can be deduced from Theorem B and Theorem C. In fact, they are direct consequences of Theorem $\mathrm{C}$ and the following result:

THEOREM 4. Let $f$ be a finite function defined on the real line $R$. For the function $f$ to be an exact generalized Peano derivative on $R$ it is necessary and sufficient that there exists a distribution $T$ of finite order such that the value $v(T, x)$ exists for all $x$ in $R$ and $v(T, \cdot)=f(\cdot)$ on $R$.

Proof. (i) Necessary condition. Suppose that the finite function $f$ is the exact generalized $n$th Peano derivative of a continuous function, say $F$, on $R$. Then, the $n$th distributional derivative, denoted by $T$, of the regular distribution determined by the continuous function $F$ is of finite order (cf. [42], [34]). We show that $v(T, x)$ exists and is equal to $f(x)$ for all $x$ in $R$. To this end, let $x_{0} \in R$ be fixed. Since $F_{[n]}\left(x_{0}\right)=f\left(x_{0}\right)$, there exists a nonnegative integer $k=k\left(x_{0}\right)$ such that $G_{n+k}\left(x_{0}\right)=$ $f\left(x_{0}\right)$, where $G=F^{-k}$ on $R$. Define $Q$ by

$$
Q(x)=G(x)-G\left(x_{0}\right)-\sum_{i=1}^{n+k-1} \frac{\left(x-x_{0}\right)^{i}}{i !} G_{i}\left(x_{0}\right)
$$

for all $x \in R$. Then, the $(n+k)$ th distributional derivative of $Q$ is just the distribution $T$, and

$$
f\left(x_{0}\right)=G_{n+k}\left(x_{0}\right)=Q_{n+k}\left(x_{0}\right)=\lim _{x \rightarrow x_{0}} \frac{(n+k) ! Q(x)}{\left(x-x_{0}\right)^{n+k}} .
$$

Hence it follows from Theorem B that $v\left(T, x_{0}\right)=f\left(x_{0}\right)$, completing the proof.

(ii) Sufficient condition. Suppose that $T$ is a distribution of finite order on $R$ such that $v(T, x)=f(x)$ for all $x \in R$. Since $T$ is of finite order, there exist (cf. [42], [34]) a continuous function $F$ on $R$ and a positive integer $n$ such that $T$ is just the $n$th distributional derivative of $F$ on $R$. We want to show that $F_{[n]}(x)$ exists and is equal to $f(x)$ for all $x \in R$. To this end, let $x_{0} \in R$ be fixed. Since $v\left(T, x_{0}\right)=f\left(x_{0}\right)$, by Theorem B there exists a function $G$ continuous in a neighborhood $N$ of $x_{0}$ and there exists a nonnegative integer $m$ such that the $m$ th distributional derivative of $G$ is equal to $T$ on $N$ and

$$
\lim _{x \rightarrow x_{0}} \frac{G(x)}{\left(x-x_{0}\right)^{m}}=\frac{f\left(x_{0}\right)}{m !} .
$$

The last equation means that the $m$ th Peano derivative of $G$ at $x_{0}$ exists and is equal to $f\left(x_{0}\right)$, i.e. $G_{m}\left(x_{0}\right)=f\left(x_{0}\right)$. Now, since the $n$th distributional derivative of $F$ and the $m$ th distributional derivative of $G$ are equal on $N$, one concludes that on $N$ one of the functions $F$ and $G$ is a $|m-n|$ th fold integral of the other. If $m>n$, then $G_{m}\left(x_{0}\right)=f(x)$ means that $F_{[n]}\left(x_{0}\right)=f\left(x_{0}\right)$. If $m<n$ then $G_{m}\left(x_{0}\right)=f\left(x_{0}\right)$ implies that $F_{n}\left(x_{0}\right)=f\left(x_{0}\right)$ by supplying l'Hôpital rule $(n-m)$ times. The proof is hence completed.

Should the distribution $T$ be not necessarily of finite order, one has the following:

Corollary 1. If the value, $v(T, x)$, of a distribution $T$ exists for all $x$ in $R$, then $v(T, \cdot)$ is an exact generalized Peano derivative on each bounded interval. 
COROllary 2. If $f$ is a pointwisely Cesàro continuous function on $R$, then there exists a distribution $T$ on $R$ such that the value, $v(T, x)$, exists and is equal to $f(x)$ for all $x$ on $R$.

3. Generalizations of integrals. The integrals of Perron type to be discussed later on are based on the following monotonicity theorems:

TheOREM D [4, TheOREM 5]. Let $F$ be a finite function satisfying the following conditions on a compact interval I:

(1) $F$ is in Baire class one;

(2) $F$ has the Darboux property;

(3) the approximate derivative, $D_{\mathrm{ap}} F$, exists (finite or infinite) except perhaps on a countable set (i.e. nearly everywhere);

(4) $D_{\text {ap }} F \geqslant 0$ almost everywhere.

Then $F$ is continuous and monotone increasing on $I$.

Theorem E [45, TheOrem 3.2]. Let $F$ be a finite function satisfying the following conditions on an open interval 1 :

(1) $F$ is in Baire class one;

(2) $F$ had the Darboux property;

(3) $l D_{s} F \geqslant 0$ almost everywhere, and $l D_{s} F>-\infty$ everywhere.

Then $F$ is monotone increasing on $I$.

Here, $l D_{s} F(x)$ denotes the lower symmetric derivate of the function $F$ at $x$ and is defined by

$$
l D_{s} F(x)=\liminf _{h \rightarrow 0}[F(x+h)-F(x-h)] / 2 h .
$$

The meanings of $u D_{s} F(x), D_{s} F(x)$ should be clear. If the lim inf above is replaced by the approximate lim inf, one has the lower approximate symmetric derivate $l D_{\text {sap }} F(x)$, and then $u D_{\text {sap }} F(x)$ and $D_{\text {sap }} F(x)$, etc.

We remark that the condition (3) in Theorem $\mathrm{E}$ is a little weaker than the condition " $l D_{s} F(x) \geqslant 0$ for all $x$ on $I$," originally assumed by Weil [45]. That the same conclusion holds under the weaker condition here can be proved by using a standard procedure (cf. [27]). It would be interesting to know whether Theorem E holds true in case the symmetric derivate $l D_{s} F$ there is replaced by the approximate symmetric derivate $l D_{\text {sap }} F$.

TheOREM F [29, THEOREM 1]. For a finite function $F$ to be monotone increasing on a compact interval $I$ it is necessary and sufficient that the following conditions hold:

(1) $F$ is upper closed monotone on I or simply F is uCM on I (i.e. F is monotone increasing on the closure of any open subinterval of $I$ whenever it is so on the open subinterval);

(2) $F$ is generalized lower absolutely continuous on I, or simply $F$ is [lACG] on I (i.e. I can be written as a union of countably many closed sets on each of which the function $F$ is lower (semi-) absolutely continuous);

(3) the upper derivate $u F_{1} \geqslant 0$ almost everywhere on $I$. 
As in [29], a class of functions, say $\mathcal{F}$, is termed an upper (semi-) linear space if the class is closed under linear combinations with nonnegative coefficients, i.e. $\mathscr{F}$ contains $\alpha f+\beta g$ whenever $f, g$ are in $\mathscr{F}$ and $\alpha, \beta$ are nonnegative constants. "Dually," $\mathscr{F}$ is a lower linear space if $-\mathscr{F}=\{f:-f \in \mathcal{F}\}$ is an upper linear space. It is clear that a class of functions is a linear space if and only if the class is both an upper and lower linear space. Note that each of the classes $C_{n}, C_{\infty}, p C_{\infty}, G_{n} P D$, $G P D$ is a linear space; the class of all the [lACG] functions is an upper linear space; but neither the class of all $u C M$ functions nor the class of all the Darboux functions which are in Baire class one is a semilinear space (see [5], where many references on Baire one Darboux functions can be found).

Throughout the rest of the section, let $u L$ be an upper semilinear space of finite functions. Using $u L$, six generalized Perron integrals, denoted as LPG $(k)$-integral for $k=1,2,3,4,5,6$, are to be defined and discussed. The development of the integrals is based on Theorem $\mathrm{F}$ for $k=1,2,3$, on Theorem $\mathrm{D}$ for $k=4,5$, and on Theorem $\mathrm{E}$ for $k=6$. Thus, for the first three integrals, the space $u L$ is assumed to be contained in the class of all $u C M$ functions; and for the last three integrals, $u L$ is assumed to be contained in the class of all Baire one Darboux functions. (Note that every Darboux function on an interval is $u C M$ there.) Furthermore, for the integrals to be general enough, we also assume that $u L$ contains all continuous functions. A "principle" is that the larger the upper linear space $u L$ is the more general the resulting integrals are.

Let $f$ be a function which is finite almost everywhere on a compact interval $I=[a, b], a<b$. A function $M$ is called a $\operatorname{LPG}(k)$-major function of $f$ on $I$ if $M$ is in $u L, M(a)=0$ and if the conditions $(\alpha, k)$ and $(\beta, k)$ are satisfied:

$(\alpha, k) M$ is $[l A C G]$ on $I$ for $k=1,2,3$.

$(\alpha, 4) D_{\text {ap }} M(x)$ exists (finite or infinite) and is $>-\infty$ for nearly all $x$ in $I$.

$(\alpha, 5) D_{\text {ap }} M(x)$ exists (finite or infinite) and is $<+\infty$ for nearly all $x$ in $I$.

$(\alpha, 6) l D_{s} M(x)>-\infty$ for all $x$ in $I$.

$(\beta, k) l \mathscr{D}_{k} M(x) \geqslant f(x)$ for almost all $x$ in $I$, where

$$
l \mathscr{D}_{k} M(x)= \begin{cases}l D_{\mathrm{ap}} M(x) & \text { for } k=1,4,5, \\ l D_{s} M(x) & \text { for } k=3,6, \\ l D_{\text {sap }} M(x) & \text { for } k=2 .\end{cases}
$$

A function $m$ is called a $\operatorname{LPG}(k)$-minor function of $f$ on $I$ if $-m$ is a LPG $(k)$-major function of $-f$ on $I$.

Fundamental to the development of the theory of LPG $(k)$-integral is the following result for $k=1,2,3,4,5,6$.

LEMMA $(2, k)$. If $M$ is a LPG( $k)$-major function and $m$ is a LPG $(k)$-minor function of the same function $f$ on $I$, then $M-m$ is nonnegative and monotone increasing on $I$.

Proof. Since $M(a)-m(a)=0$, it suffices to show that $M-m$ is monotone increasing on $I$. Writing $F=M-m$, one sees that $F$ is in $u L$ since $u L$ is an upper linear space. Furthermore, it is easy to check that $F$ satisfies all the conditions in Theorem $\mathrm{F}$ for $k=1,2,3$, in Theorem $\mathrm{D}$ for $k=4,5$, and in Theorem $\mathrm{E}$ for $k=6$. Hence we see that $F$ is monotone increasing on $I$. 
Now, let

$$
\begin{aligned}
& u \operatorname{LPG}(k) \int_{a}^{b} f(x) d x=\inf M(b), \\
& u \operatorname{LPG}(k) \int_{a}^{b} f(x) d x=\sup m(b),
\end{aligned}
$$

where the inf (sup, resp.) is taken over all the LPG $(k)$-major functions $M$ (minor functions $m$, resp.) of $f$ on $[a, b]$. The above two numbers (perhaps infinite) are, respectively, called the upper and lower LPG $(k)$-integrals of $f$ on $[a, b]$. If these two upper and lower integrals are equal, the common value, denoted as $\operatorname{LPG}(k) \int_{a}^{b} f(x) d x$, is called the LPG $(k)$-integrals of $f$ on $[a, b]$. Should the LPG $(k)$ integral of $f$ on $[a, b]$ exist and be finite, the function $f$ is said to be LPG $(k)$-integrable on $[a, b]$. Note that it follows easily from Lemma $(2, k)$ that both the upper and lower LPG $(k)$-integrals of $f$ are finite if and only if the function $f$ has both LPG $(k)$-major and minor functions. We list some properties of the integrals, the proofs of which, essentially based on Lemma $(2, k)$ are routine and are omitted here (cf. [38], [8]).

(i) For the function $f$ to be $\operatorname{LPG}(k)$-integrable on $[a, b]$ it is necessary and sufficient that for each positive member $\varepsilon$ there exist a $\mathrm{LPG}(k)$-major function $M$ and a LPG $(k)$-minor function $m$ of $f$ on $[a, b]$ such that $M(b)-m(b)<\varepsilon$.

(ii) If $f$ is LPG $(k)$-integrable on $[a, b]$, then $f$ is also LPG $(k)$-integrable on $[a, x]$ for all $x \in(a, b)$.

(iii) The class of all the $\operatorname{LPG}(k)$-integrable functions on $[a, b]$ is a linear space and the $\operatorname{LPG}(k)$-integral is a linear function on the space.

(iv) Let $f$ be $\operatorname{LPG}(k)$-integrable on $[a, b]$ and let

$$
F(x)= \begin{cases}\operatorname{LPG}(k) \int_{a}^{x} f(t) d t & \text { for } x \in(a, b], \\ 0 & \text { for } x=a .\end{cases}
$$

Then there exist a sequence $\left\{M_{n}\right\}$ of LPG $(k)$-major functions $M_{n}$ and a sequence $\left\{m_{n}\right\}$ of LPG $(k)$-minor functions $m_{n}$ of $f$ on $[a, b]$ such that $M_{n} \rightarrow F$ and $m_{n} \rightarrow F$ uniformly on $[a, b]$.

(v) Let $f$ and $F$ be as in (iv). Then $M-F$ and $F-m$ are monotone increasing on $[a, b]$ for any LPG $(k)$-major function $M$ and any LPG $(k)$-minor function $m$ of $f$ on $[a, b]$.

(vi) Let $f$ and $F$ be as in (iv). Then both $F$ and $-F$ are in $u L$ provided that the upper linear space $u L$ is closed under uniform convergences on $[a, b]$.

(vii) Let $f$ and $F$ be as in (iv). Then the "derivative" $\mathscr{D}_{k} F(x)$ exists and is equal to $f(x)$ for almost all $x$ in $[a, b]$. (Here the meaning of the "derivative $\mathscr{D}_{k} F(x)$ ") should be clear by looking at the meaning of the lower derivate $l \mathscr{D}_{k} F(x)$ given in the condition ( $\beta, k)$ for LPG $(k)$-major functions.)

Now we list some properties related to the condition $(\alpha, k)$ as theorems below.

TheOREM $(5, k)(k=1,2,3$, ONLY). Let $f$ be $\operatorname{LPG}(k)$-integrable on $[a, b]$ and $F$ be the indefinite LPG $(k)$-integral as defined in (iv). Then both $F$ and $-F$ are [lACG] on $[a, b]$. 
Proof. Note that the LPG(1)-integral is just the LPG-integral given in [29], and hence Theorem $(5,1)$ here is just the lemma in [29]. The proof of Theorem $(5, k)$ for $k=2,3$ follows the same line as the proof of the lemma in [29] and is omitted here.

Now, for convenience, let $u A(k)$ denote the class of all functions $M$ satisfying the condition $(\alpha, k)$. Then $u A(k)$ is an upper linear space. Let

$$
\begin{aligned}
L & =\{F: F \text { and }-F \text { are in } u L\}, \\
A(k) & =\{F: F \text { and }-F \text { are in } u A(k)\} .
\end{aligned}
$$

Then both $L$ and $A(k)$ are linear spaces. Now, we are in a position to state a simple result:

THEOREM $(6, k)$. If $F$ is in $L \cap A(k)$ and if $\mathscr{D}_{k} F(x)$ exists and is finite for almost all $x$ in $[a, b]$, then $\mathscr{D}_{k} F$ is $\mathrm{LPG}(k)$-integrable on $[a, b]$ and $\mathrm{LPG}(k)-$ $\int_{a}^{b} \mathscr{D}_{k} F(x) d x=F(b)-F(a)$.

Proof. It is trivial since $F-F(a)$ serves both as a $\mathrm{LPG}(k)$-major and a LPG $(k)$-minor function of $\mathscr{D}_{k} F$ on $[a, b]$.

It is not true in general that an indefinite LPG $(k)$-integral must be in $L \cap A(k)$. However, it follows from Theorem $(5, k)$ and properties (vi) and (vii) we have the following result.

THEOREM $(7, k)(k=1,2,3 \mathrm{ONLY})$. Let $u L$ be closed under uniform convergences on $[a, b]$. Then for the function $f$ (finite almost everywhere) to be $\mathrm{LPG}(k)$-integrable on $[a, b]$ it is necessary and sufficient that there exists a function $F$ in $L \cap A(k)$ such that $\mathscr{D}_{k} F(x)=f(x)$ for almost all $x$ in $[a, b]$.

Remark 3. Ellis [18] has defined integrals of descriptive Denjoy type using $P$-classes. Following his idea, integrals of descriptive Denjoy types can be defined by using the linear space $L \cap A(k)$ and the "derivative operator" $\mathscr{D}_{k}$. It follows from Theorem $(6, k)$ and Theorem $(7, k)$ that integrals obtained in this manner are at most as general as the LPG $(k)$-integrals of Perron type considered here (cf. the introduction).

Note that, in general, the LPG(2)-integral is more general than both the LPG(1)and LPG(3)-integrals. However, we have the following:

THEOREM 8. Suppose that $u L$ is closed under uniform convergences on $[a, b]$. Then the LPG(1)- and LPG(2)-integrals are equivalent on $[a, b]$.

Proof. This follows from Theorem $(7, k)$ for $k=1,2$, and the fact that each function $F$ in $A(1)=A(2)$ is approximately differentiable almost everywhere on $[a, b]$ and $D_{\text {ap }} F(x)=D_{\text {sap }} F(x)$ whenever $F$ is approximately differentiable at $x$.

TheOREM $(9, k)(k=3,6$ ONLY). Suppose that all the functions in $u L$ are measurable. Then each indefinite LPG $(k)$-integral $F$ is differentiable (in the ordinary sense) almost everywhere.

Proof. By (vii) one knows that the symmetric derivative $D_{s} F(x)$ exists and is finite for almost all $x$. Then by Theorem 1 in [32] (for $k=1$ there), one concludes that $F$ is differentiable almost everywhere. 
COROLlary 1. Suppose that all functions in $u L$ are measurable and that $u L$ is closed under uniform convergences on $[a, b]$. Then a function $f$ is LPG(3)-integrable on $[a, b]$ if and only if there exists a function $F \in L \cap A(3)$ such that the ordinary derivative $D F(x)$ exists and is equal to $f(x)$ for almost all $x$ in $[a, b]$.

COROLlary 2. Suppose that all functions in $u L$ are measurable. Then the LPG(2)integral is strictly more general than the LPG(3)-integral.

Proof. It is clear from the definition that the LPG(2)-integral is more general than the LPG(3)-integral. To show that it is strictly more general, note that there exists (see [38]) a continuous function $F$ which is in $A(3)$ but is not differentiable (in the ordinary sense) on a set of positive measures. Then by Theorem $(6, k)$ for $k=1$ that the approximate derivative, $D_{\text {ap }} F$, of the continuous $A(3)$ function $F$ is LPG(1)- (and hence is LPG(2)-) integrable with $F$ as an indefinite LPG(2)-integral. If $D_{\text {ap }} F$ were also LPG(3)-integrable with $G$ as an indefinite LPG(3)-integral, then $F$ and $G$ would differ by at most a constant term, and hence $G$ would not be differentiable (in the ordinary sense) on a set of positive measures, contradicting Theorem $(9,3)$.

The following result shows that the lower symmetric derivates in the definition of the LPG(6)-integral can be replaced by the symmetric derivatives.

THEOREM 10. For a function $f$ (finite almost everywhere) to be LPG(6)-integrable on $[a, b]$ it is necessary and sufficient that for each positive number $\varepsilon$ there exist $a$ LPG(6)-major function $M$ and $a$ LPG(6)-minor function $m$ such that $M(6)-m(b)$ $<\varepsilon$ and the symmetric derivatives $D_{s} M(x)$ and $D_{s} m(x)$ exist for all $x$ in $[a, b]$, and $D_{s} M(x) \geqslant f(x) \geqslant D_{s} m(x), D_{s} M(x) \neq-\infty, D_{s} m(x) \neq+\infty$ for all $x$ in $[a, b]$. (Note that the symmetric derivates or derivatives at the end point $a, b$ should be interpreted as ordinary one-sided ones.)

Proof. That the condition is sufficient follows from property (i). That the condition is necessary can be proved by following the line of proof of Theorem 5 in [8] and we omit it.

On the other hand, the approximate derivatives in the definition of the LPG(4)integral can be replaced by the approximate derivates under certain situations:

THEOREM 11. Suppose that the upper linear space $u L$ has the property

$(\gamma) F$ is monotone increasing on $[a, b]$ whenever $F$ is in $u L, l D_{\text {ap }} F>0$ almost everywhere and $D_{\mathrm{ap}} F>-\infty$ nearly everywhere on $[a, b]$.

Then a function $f$ is LPG(4)-integrable on $[a, b]$ if and only if for each $\varepsilon>0$ there exist a function $M$ and a function $m$ such that $M$ and $-m$ are in $u L, M(a)=m(a)$ $=0, l D_{\text {ap }} M(x)>-\infty, u D_{\text {ap }} m(x)<+\infty$ for nearly all $x$ in $[a, b], l D_{\text {ap }} M(x)>$ $f(x) \geqslant u D_{\text {ap }} m(x)$ for almost all $x$ in $[a, b]$, and $M(b)-m(b)<\varepsilon$ (for a proof, cf. [8, Theorem 5]).

REMARK 4. We do not know whether the condition " $l D_{s} F(x)>-\infty$ for all $x$ " in Theorem E can be weakened to " $l D_{s} F(x)>-\infty$ for nearly all $x$." Should the conclusion of Theorem $E$ hold true even under this weaker condition, one could replace the condition $(\alpha, 6)$ by " $l D_{s} M(x)>-\infty$ for nearly all $x$," and obtain a 
modified LPG(6)-integral, and such a modified LPG(6)-integral would have a result like Theorem 10 except that "for all" there should then be replaced by "for nearly all", just as that in the case of the LPG(4)-integral in Theorem 11.

REMARK 5. We do not know in general what concrete spaces $u L$ have the property $(\gamma)$. However, if $u L$ is the space of all the approximately continuous functions, then $u L$ has the property $(\gamma)$. This follows easily from Theorem $F$ and the following result, of which a proof is given here to show how the approximate continuity is involved.

THEOREM G (CF. [37]). Let $F$ be an approximately continuous function on $[a, b]$ and $l D_{\text {ap }} F(x)>-\infty$ for nearly all $x$ in $[a, b]$. Then $F$ is $[l A C G]$ on $[a, b]$.

Proof. Let $A$ be the set of all points $x$ in $[a, b]$ such that $l D_{\text {ap }} F(x)>-\infty$. Then for each point $x \in A$ we can choose a positive integer $n$ such that the set $\{y \mid(F(y)-F(x)) /(y-x) \leqslant-n\}$ has $x$ as a point of dispersion. Hence, denoting by $A_{n}$ the set of points $x \in A$ such that the inequality $0 \leqslant h<1 / n$ implies both the inequalities

$$
\begin{aligned}
& \mid\{y \mid F(y)-F(x) \leqslant-n(y-x) \text { and } x \leqslant y \leqslant x+h\} \mid \leqslant h / 3, \\
& \mid\{y \mid F(x)-F(y) \leqslant-n(x-y) \text { and } x-h<y<x\} \mid \leqslant h / 3,
\end{aligned}
$$

we have $A=\cup{ }_{n=1}^{\infty} A_{n}$. Denoting further, for each integer $i$, by $A_{n}^{i}$ the part of $A_{n}$ contained in $[i / n,(i+1) / n]$, we obtain $A=\cup_{n=1}^{\infty}\left(\cup_{i=-\infty}^{\infty} A_{n}^{i}\right)$.

Consider now any two points $x_{1}, x_{2}$ in $A_{n}^{i}$ with $x_{1}<x_{2}$. Then, by (2) and (3), there exists a point $y_{0}$ in $\left[x_{1}, x_{2}\right]$ such that the two inequalities hold:

$$
\begin{aligned}
& F\left(y_{0}\right)-F\left(x_{1}\right)>-n\left(y_{0}-x_{1}\right), \\
& F\left(x_{2}\right)-F\left(y_{0}\right)>-n\left(x_{2}-y_{0}\right)
\end{aligned}
$$

so that

$$
F\left(x_{2}\right)-F\left(x_{1}\right)>-n\left(x_{2}-x_{1}\right) \text {. }
$$

The last inequality is thus established for all pairs of points $x_{1}, x_{2}$ in $A_{n}^{i}$ with $x_{1}<x_{2}$, and then it follows at once that the function $F$ is lower (semi-) absolutely continuous on $A_{n}^{i}$ (i.e. $F$ is $l A C$ on $A_{n}^{i}$ ).

It will next be shown that $F$ is lower (semi-) absolutely continuous on the closure of $A_{n}^{i}$, which will be denoted as $B_{n}^{i}$. To this end, let $\varepsilon>0$ be given. Since $F$ is $l A C$ on $A_{n}^{i}$, we can fix a positive number $\delta$ with $\delta<\varepsilon / 24 n$ and such that

$$
\sum\left[F\left(x_{j}^{\prime}\right)-F\left(x_{j}\right)\right]>-\varepsilon
$$

whenever $\left\{\left[x_{j}, x_{j}^{\prime}\right]\right\}$ is any set of nonoverlapping intervals with end points $x_{j}, x_{j}^{\prime}$ in $A_{n}^{i}$ and $\Sigma\left(x_{j}^{\prime}-x_{j}\right)<\delta$. Now, let $\left\{\left[t_{j}, t_{j}^{\prime}\right]\right\}$ be any finite set of nonoverlapping intervals with end points $t_{j}, t_{j}^{\prime}$ in $B_{n}^{i}$ and $\sum\left(t_{j}^{\prime}-t_{j}\right)<\delta$. The set $\left\{\left[t_{j}, t_{j}^{\prime}\right]\right\}$ can be reduced to a set in which the intervals have no end points in common without changing the sum $\Sigma\left[F\left(t_{j}^{\prime}\right)-F\left(t_{j}\right)\right]$, and from the resulting set all intervals with both end points in $A_{n}^{i}$ can be dropped without decreasing the resulting sum $\Sigma\left[F\left(t_{j}^{\prime}\right)-F\left(t_{j}\right)\right]$ by more than $\varepsilon$. Thus, we can assume that the intervals in the set $\left\{\left[t_{j}, t_{j}^{\prime}\right]\right\}$ have no end points in common and at least one of the end points of each interval is in $B_{n}^{i}$ but not $A_{n}^{i}$. Let $\left[t_{j}, t_{j}^{\prime}\right]$ be such that $t_{j} \in A_{n}^{i}, t_{j}^{\prime} \in B_{n}^{i} \sim A_{n}^{i}$ and with 
$t_{j}^{\prime}$ a limit point of $A_{n}^{i}$ on the left. There are seven other possibilities. Fix $t_{j}^{0} \in A_{n}^{i}$ with $t_{j}<t^{0}<t_{j}^{\prime}$ and $t_{j}^{\prime}-t_{j}^{0}<1 / n$, and such that for arbitrary $\varepsilon_{j}>0$, the inequality holds

$$
\mid\left\{y|| F(y)-F\left(t_{j}^{\prime}\right) \mid>\varepsilon_{j} \text { and } t_{j}^{0} \leqslant y \leqslant t_{j}^{\prime}\right\} \mid<\left(t_{j}^{\prime}-t_{j}\right) / 3 \text {. }
$$

This is possible because $F$ is approximately continuous at $t_{j}^{\prime}$. It then follows from (2) and (4) that there exists a point $y_{j}^{0}$ in $\left[t_{j}^{0}, t_{j}^{\prime}\right]$ such that the following two inequalities hold

$$
\begin{aligned}
& F\left(y_{j}^{0}\right)-F\left(t_{j}^{0}\right)>-n\left(y_{j}^{0}-t_{j}^{0}\right), \\
& F\left(t_{j}^{\prime}\right)-F\left(y_{j}^{0}\right)>-\varepsilon_{j},
\end{aligned}
$$

so that $F\left(t_{j}^{\prime}\right)-F\left(t_{j}^{0}\right)>-n\left(y_{j}^{0}-t_{j}^{0}\right)-\varepsilon_{j}$. Hence

$$
\begin{aligned}
F\left(t_{j}^{\prime}\right)-F\left(t_{j}\right) & =\left[F\left(t_{j}^{\prime}\right)-F\left(t_{j}^{0}\right)\right]+\left[F\left(t_{j}^{0}\right)-F\left(t_{j}\right)\right] \\
& >-n\left(y_{j}^{0}-t_{j}^{0}\right)-\varepsilon_{j}+\left[F\left(t_{j}^{0}\right)-F\left(t_{j}\right)\right] .
\end{aligned}
$$

There is a similar result for each of the other seven types of intervals. Hence if $\varepsilon_{j}$ is chosen to be so small that $-8 \Sigma \varepsilon_{j}>-\varepsilon / 3$ we easily see that

$$
\sum\left[F\left(t_{j}^{\prime}\right)-F\left(t_{j}\right)\right]>-8 n(\varepsilon / 24 n)-\varepsilon / 3-\varepsilon / 3=-\varepsilon .
$$

Thus, we have shown that $F$ is $l A C$ on $B_{n}^{i}$.

Now, list the countably many collection $\left\{B_{n}^{i} \mid n=1,2,3, \ldots, i=\right.$ $0, \pm 1, \pm 2, \ldots\}$ as $\left\{C_{k} \mid k=1,2,3, \ldots\right\}$ and let $\left\{y_{k} \mid k=1,2,3, \ldots\right\}$ be the set of points $x$ in $[a, b]$ at which $l D_{\text {ap }} F(x)>-\infty$ fails to hold, and let

$$
E_{k}=C_{k} \cup\left\{y_{k}\right\} \text { for } k=1,2,3, \ldots
$$

Then $[a, b]=\cup_{k=1}^{\infty} E_{k}$, and each $E_{k}$ is closed and the function $F$ is $l A C$ on each $E_{k}$. Thus $F$ is $[l A C G]$ on $[a, b]$, completing the proof.

Before stating a direct consequence of Theorem $G$, let us recall that we have discussed in $\$ 2$ the following classes of functions: $C_{n}, C_{\infty}, p C_{\infty}, G_{n} P D, G P D$. Furthermore, let

$$
A_{0}=\text { the class of all approximately continuous functions, }
$$

and for $n \geqslant 1$ let

$$
A_{n}=\text { the class of all exact } n \text {th approximate Peano derivatives. }
$$

Note that each of the above classes is a linear space, and each function in any of the spaces is in Baire class one and has the Darboux property on the interval concerned. Thus, taking any one of the spaces as $u L$, the $\operatorname{LGG}(k)$-integral is defined for all values of $k$, i.e. for $k=1,2,3,4,5,6$. If we take $u L=C_{n}$ (so that $\left.L=C_{n}=u L\right)$, then the resulting $\operatorname{LPG}(k)$-integral will simply be denoted as $C_{n}(k)$-integral. Similarly, the meaning of each of the following integrals should be clear: $C_{\infty}(k), p C_{\infty}(k)-, G_{n} P D(k)-, G P D(k)-, A_{n}(k)$-integrals.

Now, we have as a corollary of Theorem $\mathrm{G}$ and Theorem 11 the following result:

Corollary. The $C_{0}(1)-\left(A_{0}(1)-\right.$, respectively) integral is more general than the $C_{0}(4)-\left(A_{0}(4)-\right.$, respectively) integral. 
REMARK 6. We do not know in general whether the LPG(1)-integral is more general than the LPG(4)-integral. This leads us to ask the following general question: What kind of Baire one Darboux functions $F$ have the property that $l D_{\text {ap }} F(x)>-\infty$ for nearly all $x$ in $[a, b]$ implies that $F$ is $[l A C G]$ on $[a, b]$ ? Similarly, concerning the LPG(3)- and LPG(6)- integrals, we ask: What kind of Baire one Darboux functions $F$ have the property that $l D_{s} F(x)>-\infty$ for all (or nearly all) $x$ in $[a, b]$ implies that $F$ is $[l A C G]$ on $[a, b]$ ? At this point, we mention the following result which is somewhat parallel to Theorem $G$ and is proved in [21].

TheOREM H (CF. [21]). Let $F$ be $C_{1}$-continuous on $[a, b]$ and $l C_{1} D F(x)>-\infty$ for nearly all $x$ in $[a, b]$. Then $F$ is $[$ lACG] on $[a, b]$.

REMARK 7. It is not known whether a $C_{n}$-continuous function $F$ with $l C_{n} D F(x)$ $>-\infty$ for all $x$ in $[a, b]$ is $[l A C G]$ on $[a, b]$ when $n \geqslant 2$. However, it is known that if $F$ is $C_{n}$-continuous and $-\infty<l C_{n} D F(x) \leqslant u C_{n} D F(x)<+\infty$ for nearly all $x$ in $[a, b]$, then $F$ is $[A C G]$ on $[a, b]$ (see [39], [40] and [44]).

Now, we come to discuss some of the concrete integrals and state some of the related problems.

(a) The $C_{n}(1)$-integral is equivalent to Ellis' $G M_{n}$-integral in [16] and hence is more general than Burkill's $C_{n} P$-integral. That the $C_{n}(1)$-integral and the $G M_{n}$-integral are equivalent (cf. [29]) mainly follows from Theorem $(7,1)$ and the fact that Ellis' $M_{n}$-continuous functions on intervals are just Burkill's $C_{n}$-continuous functions (cf. [41]). Note that the space $C_{n}$ is closed under uniform convergences on compact intervals (cf. the proof of Theorem 12 at the end).

(b) The $C_{n}(1)$-integral and the $C_{n}(2)$-integral are equivalent by Theorem 8 . For $n=0$, the $C_{n}(3)$-integral "stands" between the $C_{n}(1)$ - and the $C_{n} P$-integral. This is clear by Corollary 1 to Theorem $(9,3)$ and the descriptive definitions of the $C_{0}(1)$-integral (i.e. the Denjoy-Khintchine integral in [38]) and of the $C_{0} P$-integral (i.e. the Denjoy-Perron integral in [38]). We do not know whether the $C_{n}(3)$-integral is more general than the $C_{n} P$-integral when $n \geqslant 1$.

(c) The $C_{n+1}(k)$-integral is more general than the $C_{n}(k)$-integral for all values of $n$. But we do not have this for the $A_{n+1}(k)$ - and $A_{n}(k)$-integrals (cf. [29] for $k=1$ ). This is one of the reasons that we only consider classes $C_{\infty}, p C_{\infty}, G P D$, etc., in $\$ 2$ but not $A_{\infty}, p A_{\infty}$, etc.

(d) Note that both the $C_{1} P$-integral and the $A_{0}(4)$-integral are more general than the $C_{0} P$ - (i.e. Denjoy-Perron) integral, but the $C_{1} P$ - and the $A_{0}(4)$-integrals are not compatible (cf. [19]). Letting $C_{1} P \cap A_{0}(4)=\left\{f: f\right.$ is both $C_{1} P$ - and $A_{0}$ (4)-integrable to the same value , one sees that $C_{1} P \cap A_{0}(4)$ contains at least the class of all $C_{0} P$-integrable functions. However, we do not know how to characterize the class $C_{1} P \cap A_{0}(4)$. (It seems clear that such a characterization would be closely related to the open problem 3 by Bruckner in [6].) Similar problems exist for the $C_{n+1}(k)$ and $A_{n}(k)$-integrals. We should mention that by Theorem 11 the $A_{0}(4)$-integral is "nearly" the $A P$-integral of Burkill in [10], where he required that $l D_{\text {ap }} M(x)>-\infty$ for all $x$ instead of for nearly all $x$ in the definition of his major functions. Whether the $A_{0}(4)$-integral is strictly more general than Burkill's $A P$-integral still remains to 
be seen. The $A_{0}(1)$-integral has been defined and studied by Kubota [26], and many approximately continuous integrals were studied by Ridder even earlier (cf. [37]).

(e) The classes $C_{\infty}, p C_{\infty}$ and $G P D$ seem to be new in the context of generalized integrals. The $G P D(k)$-integral is more general than the $p C_{\infty}(k)$-integral (by Corollary 2 to Theorem 1 and Definition 2), which in turn is more general than the $C_{\infty}(k)$-integral. Also we see easily that each of the $G P D(1)-, p C_{\infty}(1)$ - and $C_{\infty}(1)$-integrals is more general than Ellis $G M_{n}$ - (i.e. $\left.C_{n}(1)-\right)$ integral and hence is also more general than Burkill's $C_{n} P$-integral for all values of $n$. The $G P D(k)$-integral is more general than the $G_{n} P D(k)$-integral, which is of course more general than the $C_{n}(k)$-integral. How the $G_{n} P D(k)$-integral is related to the $A_{n}(k)$-integral needs further investigation.

(f) Many integrals of Perron type can be characterized by integrals of descriptive Denjoy type. For examples, Burkill's $C_{n} P$-integral is equivalent to Sargent's $C_{n} D$ integral (see [39] and [44],) and the LPG( $k$ )-integral is characterized in Theorem $(7, k)$ for $k=1,2,3$. However, we do not know any similar characterization for the LPG $(k)$-integral when $k=4,5$, or 6 even in the simplest case when $u L=C_{0}$.

RemarK 8. In Theorem $(7, k)$, we do not know whether the hypothesis that the space $u L$ be closed under uniform convergences is superfluous. We end this paper by proving that the space $p C_{\infty}$ is closed under uniform convergences. The same is true for the space $C_{n}, C_{\infty}$. For other spaces like $G_{n} P D, G P D$ etc., further investigation is required.

THEOREM 12. Let $f_{n}$ be a pointwisely Cesàro continuous function on $[a, b]$ for each integer $n \geqslant 1$, and let $f$ be a function such that $f_{n}$ converges to $f$ uniformly on $[a, b]$. Then $f$ is also pointwisely Cesàro continuous on $[a, b]$.

Proof. Let $c$ be a point in $[a, b]$. We want to show that $f$ is Cesàro continuous at $c$. To this end, let $\varepsilon>0$ be fixed but arbitrary. Choose $n$ such that

$$
\left|f_{n}(x)-f(x)\right|<\varepsilon / 3 \text { for all } x \in[a, b] .
$$

Note that, as a limit of Baire one functions, $f$ is also measurable. Thus, the above inequality implies that the measurable function $f_{n}-f$ is Lebesgue integrable and hence is $C_{k} P$-integrable on $[a, b]$ for any nonnegative integer $k$. Now, at the point $c$, the function $f_{n}$ is $C_{m}$-continuous for a positive integer $m(=m(c))$. Thus for $h$ sufficiently small, one has

$$
\left|C_{m}\left(f_{n} ; c, c+h\right)-f_{n}(c)\right|<\varepsilon / 3 .
$$

Furthermore, in a neighborhood of $c$, both the functions $f_{n}-f$ and $f_{n}$ are $C_{m-1} P$ integrable, and hence so is the function $f\left[=f_{n}-\left(f_{n}-f\right)\right]$. Thus, for $h$ sufficiently small, one has

$$
\begin{aligned}
\mid C_{m}(f ; c & , c+h)-f(c) \mid \\
& \leqslant\left|C_{m}\left(f_{n}-f ; c, c+h\right)\right|+\left|C_{m}\left(f_{n} ; c, c+h\right)-f_{n}(c)\right|+\left|f_{n}(c)-f(c)\right| \\
& <\varepsilon / 3+\varepsilon / 3+\varepsilon / 3=\varepsilon .
\end{aligned}
$$

Thus, the function $f$ is $C_{m}$-continuous at $c$. 
ACKNOWLeDgement. The author would like to thank Gilbert G. Walter, who has drawn the author's attention to the papers [31], [34], and for years has been an inspiration and friend to the author, and to whom the author owes a large debt of gratitude.

\section{REFERENCES}

1. B. S. Babcock, On properties of approximate Peano derivatives, Trans. Amer. Math. Soc. 212 (1975), 279-294.

2. J. A. Bergin, A new characterization of Cesàro-Perron integrals using Peano derivatives, Trans. Amer. Math. Soc. 228 (1977), 287-305.

3. L. S. Bosanquet, A property of Cesäro-Perron integrals, Proc. Edinburgh Math. Soc. 6 (1940), $160-165$.

4. A. M. Bruckner, An affirmative answer to a problem of Zahorski and some consequences, Michigan Math. J. 13 (1966), 15-26.

5. ___ Differentiation of real functions, Springer, Berlin, 1978.

6. __ Current trends in differentiation theory, Real Analysis Exchange 5 (1979-80), 9-60.

7. P. S. Bullen, The $P^{n}$-integral, J. Austral. Math. Soc. 14 (1972), 219-236.

8. P. S. Bullen and C. M. Lee, On the integrals of Perron type, Trans. Amer. Math. Soc. 182 (1973), 481-501.

9. P. S. Bullen and S. N. Mukhopadhyay, Peano derivatives and general integrals, Pacific J. Math. 47 (1973), 43-58.

10. J. C. Burkill, The approximately continuous Perron integrals, Math. Z. 34 (1931), 270-278.

11. The Cesàro-Perron integral, Proc. London Math. Soc. (2) 34 (1932), 314-322.

12. The Cesaro-Perron scale of integration, Proc. London Math. Soc. (2) 39 (1935), 541-552.

13. G. E. Cross, The expression of trigonometric series in Fourier form, Canad. J. Math. 12 (1960), 694-698.

14. A. Denjoy, Sur l'intégration des coefficients différentials d'ordre supérieur, Fund. Math. 25 (1935), 273-326.

15. M. J. Evans, $L_{p}$ derivatives and approximate Peano derivatives, Trans. Amer. Math. Soc. 165 (1972), 381-388.

16. H. W. Ellis, Mean-continuous integrals, Canad. J. Math. 1 (1949), 113-124.

17. On the compatibility of the approximate Perron and the Cesairo-Perron integrals, Proc. Amer. Math. Soc. 2 (1951), 396-397.

18. _ Darboux properties and applications to non-absolutely convergent integrals, Canad. J. Math. 3 (1951), 471-484.

19. R. Henstock, The use of convergence factors in Ward integration, Proc. London Math. Soc. (3) 10 (1960), 107-121.

20. R. D. James, Generalized nth primitives, Trans. Amer. Math. Soc. 76 (1954), 149-176.

21. R. L. Jeffrey, Non-absolutely convergent integrals, Proc. Second. Canad. Math. Congr. (Vancouver, Canada, 1949), Univ. of Toronto Press, Toronto, 1951, pp. 93-115.

22. R. L. Jeffrey and H. W. Ellis, Cesàro totalization, Trans. Roy. Soc. Canada Sect. III (3) 36 (1942), 19-44.

23. R. L. Jeffrey and D. S. Miller, Convergence factors for generalized integrals, Duke Math. J. 12 (1945), 127-142.

24. Y. Kubota, On a characterization of the CP-integral, J. London Math. Soc. 43 (1968), 607-611.

25. The mean-continuous Perron integral, Proc. Japan Acad. 40 (1964), 171- 175.

26. __ An integral of Denjoy type. I, II, III, Proc. Japan Acad. 40 (1964), 713-717; 42 (1966), 737-742; 43 (1967), $441-444$.

27. C. M. Lee, On the approximate Peano derivatives, J. London Math. Soc. 12 (1976), 475-478.

28. __ An approximate extension of Cesàro-Perron integrals, Bull. Inst. Acad. Sinica 4 (1976), 73-82.

29. An analogue of the theorem of Hake-Alexandroff-Looman, Fund. Math. 100 (1978), 69-74.

30. Note on the oscillatory behavior of certain derivatives, Real Analysis Exchange 4 (1979), 178-183.

31. S. Kojasiewicz, Sur la valeur et la limite d'une distribution en un point, Studia Math. 16 (1957), $1-36$. 
32. J. Marcinkiewicz and Z. Zygmund, On the differentiability of functions and summability of trigonometric series, Fund. Math. 26 (1936), 1-43.

33. J. C. McGregor, An integral of Perron type, thesis, Univ. of British Columbia, Vancouver, Canada (unpublished).

34. J. Mikusinski and R. Sikorski, The elementary theory of distributions. I, Rozprawy Mat. 12 (1957), $54 \mathrm{pp}$.

35. W. H. Oliver, The exact Peano derivative, Trans. Amer. Math. Soc. 76 (1954), 444-456.

36. R. O'Malley and C. Weil, The oscillatory behavior of certain derivatives, Trans. Amer. Math. Soc. 234 (1977), 467-481.

37. J. Ridder, Ueber die gegenseitigen Beziehungen verschieder approximativ stetiger Denjoy-Perron-Integrall, Fund. Math. 22 (1934), 136-162.

38. S. Saks, Theory of integral, Monogr. Mat., no. 7, Warsaw, 1937.

39. W. L. C. Sargent, A descriptive definition of Cesàro-Perron integrals, Proc. London Math. Soc. (2) 47 (1941), 212-247.

40. On generalized derivatives and Cesàro-Denjoy integrals, Proc. London Math. Soc. (2) 52 (1951), 365-376.

41. Some properties of $C_{\lambda}$-continuous functions, J. London Math. Soc. 26 (1951), 116-121.

42. L. Schwartz, Theory of distributions, Hermann, Paris, 1950.

43. S. Verblunsky, On the Peano derivatives, Proc. London Math. Soc. (3) 22 (1971), 313-324.

44. , On a descriptive definition of Cesàro-Perron integrals, J. London Math. Soc. (2) 3 (1971), 326-333.

45. C. Weil, On properties of derivatives, Trans. Amer. Math. Soc. 114 (1965), 363-376.

46. M_ Monotonicity, convexity and symmetric derivatives, Trans. Amer. Math. Soc. 222 (1976), 225-237.

47. Z. Zahorski, Über die Menge der Punkte in welchen die Ableitung unendlich ist, Tôhoku Math. J. 48 (1941), 321-330.

48. __ Sur la première dérivée, Trans. Amer. Math. Soc. 69 (1950), 1-54.

Department of Mathematics, University of Wisconsin-Milwaukee, Milwaukee, Wisconsin 53201 